\title{
Physician Responses to the \\ Opioid Epidemic in the Context of \\ Population-Based Pain and Opioid Care
}

AJAY D. WASAN

University of Pittsburgh Medical Center, University of Pittsburgh School of Medicine

The opioid epidemic is a multifaceted phenomenon in which the prescription of opioids for pain still plays a significant role. However, a plethora of efforts in the physician community following many years of epidemiological research regarding patterns and characteristics of prescription opioid misuse, abuse, and addiction have led to best practice guidelines agreed upon widely. Adoption of these guidelines and specific changes across health care systems and in individual provider practices have resulted in improved opioid prescribing patterns, which are safer and no less effective in addressing pain. The experience at the University of Pittsburgh and the University of Pittsburgh Medical Center is highlighted as an example of progressive change to address the simultaneous epidemics of poorly controlled chronic pain affecting 100 million adults in this country and prescription opioid use disorders affecting 2.2 million adults.

\section{Background}

- $\mathrm{n}$ the history of medicine, most epidemics change their fundamental nature over time. For example, the HIV epidemic in the United States was spread in the 1980s through unsafe sexual practices, but later was perpetuated by the sharing of dirty needles by intravenous drug abusers. The opioid epidemic is no different-initially lying within the complex interweaving of 
yet-to-be-fully-understood sociocultural issues (Quinones 2015) and subsequently in the overlay of two public health challenges: the better treatment of chronic pain (affecting 100 million US adults, an epidemic in itself) and the appropriate prescribing of opioids for pain relief (National Academies of Sciences, Engineering, and Medicine 2017; Institute of Medicine 2011).

The opioid epidemic of the past 10 years (versus the earlier epidemic of heroin addiction in the 1970s) was triggered, to some considerable extent, by the overprescribing of prescription opioids for pain, with a subsequent shift to heroin use by people without pain (National Academies of Sciences, Engineering, and Medicine 2017). In 2015, the number of opioid deaths from illicit opioids surpassed those from prescription opioids. Approximately 2 million people have a prescription opioid use disorder, while substantially more use heroin (Guy et al. 2017). And yet, the epidemics of chronic pain and opioid use disorder continue to be entwined, as $80 \%$ of heroin users report starting with prescription opioids, and 50\% of heroin users in substance use disorder (SUD) treatment report having chronic pain (National Academies of Sciences, Engineering, and Medicine 2017).

\section{Provider Responses}

There has been a range of responses from physicians to combat the environmental supply of unnecessary opioids, and in 2011 the rates of opioid prescribing for pain began to decrease, after increasing fourfold from 1999 to 2011 (Frenk, Porter, and Paulozzi 2017). This article will summarize the responses to the opioid epidemic from those who prescribe opioids. This is referred to as the point of care of opioid prescribing, which is the most upstream part in the cascade of opioid addiction among patients with pain. The origins and sustaining factors of the epidemic are discussed in detail elsewhere in this issue of COMMONWEALTH.

The problems associated with the overprescribing of prescription opioids, primarily for chronic noncancer pain, have been described in the medical literature for over a decade through scores of reviews and research publications. There is a fundamental distinction between pain due to cancer (which is known to respond very well to opioids) and pain not due to cancer, such as chronic low back pain or pain from nerve injury (neuropathic pain).

One of the most influential reports was a review in The New England Journal of Medicine in 2003 by Ballantyne and Mao who described many of the fundamental uncertainties regarding opioid therapy for chronic noncancer pain, such as the unknown degree of efficacy, the development of tolerance (which means less pain-relieving effects of the opioid over time), and the 
emerging rates of addiction (Ballantyne and Mao 2003). Concurrently, with FDA approval in 1996 of Purdue Pharma's OxyContin for pain, prescriptions of oxycodone increased for noncancer pain, which became a widespread practice in certain parts of the country, such as Appalachia. Subsequently, reports of addiction to OxyContin and diversion, from states such as West Virginia, frequently appeared in newspapers and magazines and on television.

The medical research community took note of these issues and began studying patients who were prescribed opioids for noncancer pain and examining who was more likely to misuse opioids (such as self-medicating by taking extra for pain or concurrent use of illegal drugs), abuse them (such as mixing them with alcohol to become intoxicated), or become addicted (a total loss of control in taking opioids and an incessant drive to obtain more, regardless of the negative consequences). Misuse, abuse, and addiction lie on a continuum of severity and are labeled opioid use disorders. Misuse can lead to addiction, although the rate of progression and the incidence of progression still remain unknown.

Generally speaking, $40 \%$ of those who are prescribed opioids chronically for noncancer pain will misuse them, and 5\%-15\% will become addicted (Vowles et al. 2015). To provide some context, the rate of nonadherence to medications for other chronic diseases, such as diabetes or congestive heart failure, is also $40 \%$. For chronic pain and opioids, the problem is taking too much of the medication, and for the latter diseases, the problem is not taking the appropriate medications in the amounts and frequency prescribed. Thus, one should not be surprised at the high rates of medication nonadherence in the treatment of chronic pain. The focus on identifying the characteristics of patients who misuse or become addicted to prescription opioids resulted in the identification of several risk factors that predicted opioid misuse: a prior history of abuse or addiction to controlled substances, concurrent use of illegal drugs, a family history of addictive disorders, a history of illegal activity, and untreated depression and anxiety disorders (affective disorders) (Butler et al. 2004).

\section{Three Pillars of Assessment for Opioid Adherence (Argoff et al. 2017)}

\section{Patient Self-Report}

Standardized scales were developed that determined the likelihood of opioid misuse (such as the Current Opioid Misuse Measure). These are patient selfreport checklists of problematic behaviors, such as taking more opioids than 
prescribed or borrowing medication from others (Butler et al. 2007). Scores on these measures have provided the clinician with a probability that a patient who has been prescribed opioids will misuse or already is misusing them.

The scales were a significant advance in tracking medication adherence, but still only generated an accurate prediction roughly $66 \%$ of the time. Nevertheless, patient self-reporting of medication-taking behaviors is an important pillar of the physician assessment of medication adherence (i.e., is a patient taking the medication the way it has been prescribed, and if not, is he or she addicted?). The self-report scales are just one method of gathering adherence information from the patient, and a detailed clinical interview will achieve the same results.

\section{Urine Drug Testing}

The second pillar in assessing the likelihood that patients prescribed opioids will misuse them, or in monitoring adherence, is urine drug screening or urine drug testing (UDT). Drug toxicology technology advanced in the 1990s to give rise to commercially available quantitative urine drug-testing platforms, first known as the gas chromatography-mass spectroscopy method (GCMS). GCMS allowed measurement of each type of illegal drug and prescription opioid and other prescription medications in the urine with a high degree of accuracy, being able to quantify an amount as low as nanograms per milliliter for substances such as cocaine, oxycodone, morphine, and even gabapentin (a common nonopioid pain medication).

UDTs using GCMS technology revolutionized the field of medication adherence monitoring in patients with pain. In 2007, Wasan and colleagues reported the largest series to date using UDTs to monitor prescription opioid adherence in 550 patients with chronic pain (Michna et al. 2007). They confirmed the results from earlier patient self-report studies and also found a $40 \%$ incidence of opioid misuse. This study, among others, led to the rapid and widespread adoption of UDTs as a standard of care in opioid adherence monitoring.

\section{Provider Evaluation}

The third pillar of the three-legged platform for assessing opioid adherence is the global assessment of the provider-namely, is the patient taking the medication properly? This may be information gathered from other providers of the patient (such as in the electronic medical record), reports from the family, inconsistencies observed in the patient (such as requesting early refills), lost 
or stolen prescriptions, and observed behaviors during the visit (such as sedation, confusion, or intoxication).

Provider assessment was given a significant boost with the creation of prescription drug monitoring programs (PDMPs). These are state-run databases of prescriptions of controlled substances entered by each pharmacy when a medication is dispensed. When a provider checks the PDMP they can see when the last prescription (if any) for an opioid was written by any provider in that state. Patients who are "doctor shopping" by going to multiple providers to obtain opioids can be readily identified. There has been a 30\% reduction in opioids prescribed for pain in the states with robust PDMP programs (Finley et al. 2017).

Attempts to nationalize a PDMP program were thwarted by a lack of financial support from Congress. Subsequently, several states have agreed to share their PDMP data, so that a provider can check if prescriptions were filled in adjacent states. In 2016, Pennsylvania became the 49th state with a PDMP, leaving Missouri as the only state without a program. In July 2017, the Missouri governor issued an executive order establishing a state PDMP, but it has not yet been implemented. Many states, such as Pennsylvania and New York, require that providers check the PDMP prior to the prescription of opioids. For more information on PDMPs in Pennsylvania, see Mirigian et al. 2018, in this issue.

Patient self-reports, UDT results, and provider assessment are the three fundamental components of assessing adherence to prescription opioids in a comprehensive fashion. An abnormality in any of these domains indicates that the patient is misusing opioids, at the very least, and may possibly be addicted. Wasan and colleagues developed the most widely used research criteria for identifying prescription opioid misuse, known as the Drug Misuse Index (Jamison et al. 2010). The Drug Misuse Index triangulates these three streams of data to make an overall determination of adherence versus misuse. It has been used in multiple studies and is considered the leading approach for opioid medication adherence monitoring (Smith et al. 2015).

The dose of opioid prescribed was also found to be an important predictor of complications from prescription opioid therapy, such as overdose, addiction, and death (Edlund et al. 2010). Since there are many different prescription opioid compounds of various strengths (e.g., oxycodone, morphine, fentanyl), in comparing doses, conversion is made to each compound's strength relative to morphine, the first prescription opioid. This is expressed as morphine equivalent dosing. Doses greater than $200 \mathrm{mg}$ per day of morphine equivalents were found to independently predict increased rates of overdose, addiction, and death related to opioids. Subsequent work found that doses as 
low as $90 \mathrm{mg}$ per day were just as predictive, and $90 \mathrm{mg}$ became an important benchmark in determining what is a "high dose" of opioids.

However, these studies suffered from an important confounder, in that many of the patients who had complications from opioids were also coprescribed benzodiazepines, which are medications such as Valium, Ativan, or Xanax that negatively interact with opioids. These "tranquilizers" magnify the side effects of opioids, such as sedation, confusion, and difficulty breathing. When additional studies controlled for the concurrent prescribing of benzodiazepines, the association between $90 \mathrm{mg}$ per day of morphine equivalents and increased opioid complications disappeared. Nevertheless, the 90-mg-per-day benchmark has been retained in many state and national "best practices" recommendations.

Over the past 15 years, research has continued to investigate the importance of risk factors for prescription misuse, abuse, and addiction. Of the identified factors, a previous or current history of an addictive disorder is the most powerful risk factor in predicting opioid use disorders. However, affective disorders (such as major depression, generalized anxiety disorder, or the frequent combination of both-major depression with anxious features) are most prevalent in patients with chronic pain, afflicting at least $30 \%$ of the 100 million adults in this country with chronic pain.

Among those patients seeking care for chronic pain, 40\%-50\% have an untreated or undertreated negative affective disorder (Dersh et al. 2006). This subgroup with psychiatric comorbidity is also prescribed opioids for chronic pain at much greater rates, likely due to worse levels of reported pain, disability, and distress. Wasan and others across the country have studied the trajectories of opioid therapy in the subgroup of chronic low back pain patients with negative affective disorders (Braden et al. 2009; Wasan et al. 2015).

In sum, they have found that the back pain patients with high levels of negative affective symptoms have $50 \%$ less pain relief with opioids, and a $50 \%$ rate of opioid misuse. In this group of patients, the misuse phenomenon begins with urges to take more medication than prescribed between doses, a preoccupation with the next dose, and craving for more medication. Patients with high negative affect also tend to self-medicate for anxious and depressive feelings or insomnia through taking extra opioids (Martel et al. 2014). While perhaps understandable, this is considered nonadherence to opioid therapy, and overtaking opioids is the most frequent presentation of opioid misuse.

These clinical studies and continued observations of problematic opioid prescribing involving high doses to patients with risk factors for opioid complications led state and federal authorities as well as medical professional 
societies to draft best practice guidelines for opioid therapy for chronic pain. By and large, over a period of several years, these various guidelines are quite consistent, beginning with the American Pain Society-American Academy of Pain Medicine (APS/AAPM) guidelines in 2009, and most recently, the 2016 primary care opioid prescribing guidelines for chronic pain released by the Centers for Disease Control and Prevention (CDC) (Chou et al. 2009; Dowell, Haegerich, and Chou 2016). Despite having many of the same authors, including the same lead, one remarkable difference between the APS/AAPM and CDC guidelines is that the bar for acceptable evidence regarding opioid prescribing was changed (Busse, Juurlink, and Guyatt 2016; Tayeb et al. 2016).

In the APS/AAPM guidelines, studies of patients who were prescribed opioid therapy for at least three months were considered, and this led to the conclusion that, for chronic pain, there is moderate evidence that opioids can be effective. In the CDC guidelines, only studies that tracked patients after one year of treatment were considered acceptable, none of which exist. This led the $\mathrm{CDC}$ report to conclude that opioids are not effective for chronic pain, and this conclusion has dramatically affected practice across the country, leading many primary care physicians to reduce or eliminate opioid prescribing for chronic noncancer pain.

While in the treatment of chronic disease, studies of longer than one-year duration would seem the most prudent to include for consideration, there are scarcely any studies of any pain medication (e.g., anti-inflammatory agents such as naproxen) that are more than a year long, and the vast majority are less than six months in duration (Tayeb et al. 2016). If one were to apply to all pain medications the same standard of yearlong follow-up for inclusion as acceptable studies in evidence-based guidelines, at least $75 \%$ of the existing medication guidelines for pain management would be dismissed. Thus, applying a standard for acceptable evidence that is far beyond what the field of pain management considers acceptable is far outside the norm for evidence-based guidelines for any treatment for pain.

This issue of standards for evidence inclusion is the most controversial in the CDC guidelines, but the recommendations for dose limits also were quite provocative (Busse, Juurlink, and Guyatt 2016). The CDC guidelines suggested that there is no evidence that doses greater than $90 \mathrm{mg}$ of morphine equivalents per day are more effective for chronic pain, and that these doses are associated with greater complications. As noted, this supposition has weak supporting evidence; nevertheless, it has sparked many insurance companies to deny authorization for total daily doses greater than $90 \mathrm{mgs}$, even in patients who have been on higher doses for long periods of time with evidence of improved pain and function, and no misuse. 
The majority of recommendations were well received, however, being mainstream and consistent with other guidelines. The common elements among many of these guidelines are that patients should be considered for opioids for chronic pain only after a diagnosis has been made through a medical evaluation, including a detailed history, physical exam, and review of pertinent studies; merely describing a patient having pain in the right leg, for instance, is not a diagnosis. Opioids should only be considered after several nonopioid options have failed, such as anti-inflammatory agents or physical therapy. Indeed, it is frequently pointed out that many patients with musculoskeletal pain are prescribed an opioid even before physical therapy is ordered.

Opioids should be considered a trial in which the patient and provider mutually agree on benchmarks for success, such as improvements in pain and/or function, with no issues of misuse. Opioid treatment agreements are recommended to facilitate this communication and mutual expectations. Ideally, any successful treatment for chronic pain should improve pain and function together. However, it is quite common for patients to say that a treatment has indeed helped them and improved their quality of life through being able to increase their activity, while their level of pain (most commonly rated on a $0-10$ scale, with 10 being the most severe) has not changed. Hence, changes in pain and changes in function are not always linked, which is a crucial consideration in evaluating the success or failure of treatments for chronic pain.

When starting opioids, it is important to start with low doses of shortacting opioids, which typically last 3-6 hours. This strategy avoids the complications of higher doses, particularly in the elderly, and allows a window to evaluate their effectiveness and tolerability. If opioids are deemed effective, then either switching the short-acting opioids to longer-acting formulations or adding long-acting opioids to short-acting opioids is frequently recommended. This approach avoids the peaks and valleys in blood levels of medication and levels of pain related to the dosing of short-acting opioids only. A core principle of managing chronic pain is using treatments that minimize the possible spikes in pain levels that occur frequently during the day related to activity, or that occur spontaneously at night.

In terms of monitoring adherence, the guidelines are very consistent in recommending the three pillars of assessment: patient self-report, UDTs, and provider evaluation. There are no agreed-upon intervals to do each of these things (which will vary from patient to patient, ideally based on their risks of opioid misuse), but generally when starting opioids, patients are seen within the first 2-4 weeks. When stable doses have been achieved with documentation of good adherence, patients are often seen for reevaluation every 3-6 months. If opioids are discontinued, it is recommended that they are tapered 
slowly, such as a $10 \%-20 \%$ reduction every month in total daily dose, except for the scenarios of addiction to opioids or diversion.

As an illustration of opioid guideline implementation, in 2015 the University of Pittsburgh Medical Center (UPMC) began operationalizing good opioid care practices across its integrated delivery network of 25 hospitals, 400 outpatient clinics, and a health insurance plan with 1.8 million members. This was most salient in a metropolitan region noted by the CDC to be in the upper $25 \%$ in the country of rates of opioid prescribing and overdose deaths related to opioids. UPMC is the largest academic integrated delivery network in the nation, and approximately $50 \%$ of people in the Pittsburgh metropolitan area of 2.2 million people receive at least some medical care through UPMC.

Thus, the opioid epidemic was also an opportunity to do effective population-based pain care to target this intersection of pain and opioid use. A multipronged approach was taken to improve opioid care by following the 2009 APS/AAPM prescribing guidelines for chronic pain, and scaling up/ translating the clinical implications of much of the published opioid research, such as that of Dr. Wasan, who was leading these population-based research efforts at the University of Pittsburgh.

First, a system-wide advisory committee (the Pain Steering Committee) was formed, consisting of leaders across UPMC, including several physician specialties (pain medicine, physiatry, psychiatry, primary care, and emergency medicine), nursing executives, hospital leaders, quality improvement specialists, finance administrators, and executives from the health insurance plan. The committee quickly achieved consensus that we must target the intersection between good pain care and opioid prescribing through improving pain care overall, and opioid care specifically.

We also agreed to focus on process improvements, since, like many health care systems across the country, high-quality outcomes data are still not widely available or able to be tracked sufficiently when changes to care are made. The field of medical informatics is still evolving toward this goal to enable better assessment and delivery of value-based health care. We conducted a needs assessment in multiple medical specialties regarding pain care. With the resources allotted to our committee by UPMC, we improved opioid care delivery in the following nine ways, as summarized in Table 1.

Below is more detail on some of these improvements.

1. Widely disseminating the Pennsylvania and 2016 CDC guidelines.

2. Mandating online continuing education in pain and opioid care for 500 primary care and specialty physicians along with 2,000 nurse practitioners and physician assistants. 


\begin{tabular}{|l|}
\hline Table 1. Nine Improvements to Opioid Care Delivery from UPMC \\
\hline 1. Disseminate Pennsylvania and 2016 CDC guidelines widely \\
\hline 2. Mandate online continuing education for health care professionals \\
\hline 3. Conduct intervention (90-90 Program) in select group of physicians \\
\hline 4. Provide clinical tools in electronic medical records \\
\hline 5. Promote continuing medical education opportunities \\
\hline 6. Create pathway in emergency departments for MAT/Suboxone \\
\hline 7. Embed social workers in clinics to comanage Suboxone prescribing with MDs \\
\hline 8. Collaborate and coordinate care via University of Pittsburgh Opioid Task Force \\
\hline 9. Expand multidisciplinary pain treatment resources in pain clinics \\
\hline
\end{tabular}

3. Identifying those providers who prescribed high-dose opioids for chronic pain to many patients using UPMC Health Plan data (>90 mg of morphine equivalents for $>90$ consecutive days), and then conducting an intervention in a select group of primary care physicians. This intervention (the 90-90 Program) individually mentored physicians on individual patient cases of their own to improve opioid care by slowly reducing the dose, adding nonopioid medications and physical therapy, and more tightly doing adherence monitoring. This process is known as academic detailing, with studies supporting its effectiveness in pain care specifically (Liebschutz et al. 2017). Compared to a control group of 90-90 physicians who received the guidelines but no individual mentorship, prescribed opioid doses were reduced by an average of $30 \%$ over a one-year period in the intervention group.

4. Providing a range of clinical tools in the outpatient and inpatient electronic medical records to improve pain and opioid care, such as posting of opioid guidelines, dose conversion tables, a resource list facilitating referral to pain or addiction medicine specialists, prescribing intranasal naloxone to patients who have overdosed or who are prescribed opioids and are at a higher risk of overdose, and an individual physician "opioid dashboard" enabling each physician to track across their patient panel as a whole, and for patients, individual opioid care metrics (such as opioid doses, opioid use agreements, UDTs, and compliance checklists).

5. Promoting continuing medical education opportunities to satisfy maintenance of certification requirements for internists through a monthlong project to track opioid doses and use of opioid 
agreements in their patients with chronic pain. This was completed by 300 primary care physicians.

6. Creating a pathway in the emergency departments for assessing the need for medication-assisted therapy and enabling Suboxone prescribing.

7. Embedding social workers with addiction medicine training in primary care and pain specialty clinics, along with training physicians, to prescribe Suboxone to patients with opioid use disorders, with or without pain.

8. Collaborating with addiction medicine clinicians, services, and programs, and local and regional Department of Health officials and law enforcement throughout the western Pennsylvania region via the University of Pittsburgh Opioid Task Force to better coordinate opioid addiction care.

9. Expanding multidisciplinary pain treatment resources in pain specialty clinics, such as the addition of a psychologist and a psychiatrist, and improved physical and occupational therapy services, with providers trained specifically in chronic pain rehabilitation.

\section{Conclusion}

Across the United States, the physician focus on improved opioid prescribing for chronic pain has resulted in an overall drop of $12 \%$ in the rate of opioid prescribing since 2011, decreases in the average milligrams of morphine equivalents per prescription, as well as a $30 \%$ drop in the rates of prescribing high-dose opioids, $>90 \mathrm{mg}$ per day (Guy et al. 2017). In western Pennsylvania specifically, these metrics have dropped even more sharply compared to the rest of the country (such as a 14\% drop in the rate of opioid prescribing), and as a result, our region is no longer in the upper $25 \%$ of opioid prescribing rates in the country, and is now in the middle 50\% tier (Guy et al. 2017). Local and regional efforts through UPMC and the University of Pittsburgh Opioid Task Force have certainly contributed to this success, and illustrate how population-based pain and opioid care can be translated effectively from clinical studies and guidelines to widespread clinical practice.

\section{REFERENCES}

Argoff, C. E., D. P. Alford, J. Fudin, J. A. Adler, M. J. Bair, R. C. Dart, R. Gandolfi, B. H. McCarberg, S. P. Stanos, J. A. Gudin, R. C. Polomano, and L. R. Webster. 2017. "Rational Urine Drug Monitoring in Patients Receiving Opioids for Chronic Pain: Consensus Recommendations." Pain Medicine 19 (January): 97-117. 
Ballantyne, J. C., and J. Mao. 2003. “Opioid Therapy for Chronic Pain.” The New England Journal of Medicine 349 (November): 1943-1953.

Braden, J. B., M. D. Sullivan, G. T. Ray, K. Saunders, J. Merrill, M. J. Silverberg, C. M. Rutter, C. Weisner, C. Banta-Green, C. Campbell, and M. Von Korff. 2009. "Trends in Long-Term Opioid Therapy for Noncancer Pain among Persons with a History of Depression." General Hospital Psychiatry 31 (6): 564-570.

Busse, J. W., D. Juurlink, and G. H. Guyatt. 2016. "Addressing the Limitations of the CDC Guideline for Prescribing Opioids for Chronic Noncancer Pain.” Canadian Medical Association Journal 188 (December): 1210-1211.

Butler, S. F., S. H. Budman, K. C. Fernandez, B. Houle, C. Benoit, N. Katz, and R. N. Jamison. 2007. "Development and Validation of the Current Opioid Misuse Measure." Pain 130 (July): 144-156.

Butler, S. F., S. H. Budman, K. Fernandez, and R. N. Jamison. 2004. "Validation of a Screener and Opioid Assessment Measure for Patients with Chronic Pain." Pain 112 (November): 65-75.

Chou, R., G. J. Fanciullo, P. G. Fine, J. A. Adler, J. C. Ballantyne, P. Davies, M. I. Donovan, D. A. Fishbain, K. M. Foley, J. Fudin, A. M. Gilson, A. Kelter, A. Mauskop, P. G. O'Connor, S. D. Passik, G. W. Pasternak, R. K. Portenoy, B. A. Rich, R. G. Roberts, K. H. Todd, and C. Miaskowski. 2009. "Clinical Guidelines for the Use of Chronic Opioid Therapy in Chronic Noncancer Pain.” The Journal of Pain 10 (February): 113-130.

Dersh, J., R. J. Gatchel, T. Mayer, P. Polatin, and O. R. Temple. 2006. "Prevalence of Psychiatric Disorders in Patients with Chronic Disabling Occupational Spinal Disorders." Spine 31 (May): 1156-1162.

Dowell, D., T. M. Haegerich, and R. Chou. 2016. "CDC Guideline for Prescribing Opioids for Chronic Pain-United States, 2016.” Morbidity and Mortality Weekly Report 65 (March): 1-49.

Edlund, M. J., B. C. Martin, M. Y. Fan, A. Devries, J. B. Braden, and M. D. Sullivan. 2010. "Risks for Opioid Abuse and Dependence among Recipients of Chronic Opioid Therapy: Results from the TROUP Study.” Drug and Alcohol Dependence 112 (November): 90-98.

Finley, E. P., Ashley Garcia, Kristen Rosen, Don McGeary, Mary Jo Pugh, and Jennifer Sharpe Potter. 2017. "Evaluating the Impact of Prescription Drug Monitoring Program Implementation: A Scoping Review.” BMC Health Services Research 17 (June): 420.

Frenk, S. M., K. S. Porter, and L. J. Paulozzi. 2015. "Prescription Opioid Analgesic Use among Adults: United States, 1999-2012.” NCHS Data Brief 189 (Feb): 1-8.

Guy, G. P., Jr., K. Zhang, M. K. Bohm, J. Losby, B. Lewis, R. Young, L. B. Murphy, and D. Dowell. 2017. "Vital Signs: Changes in Opioid Prescribing in the United States, 2006-2015." Morbidity and Mortality Weekly Report 66 (July): 697-704.

Institute of Medicine. 2011. Relieving Pain in America: A Blueprint for Transforming Prevention, Care, Education, and Research. Washington, DC: National Academies Press.

Jamison, R. N., E. L. Ross, E. Michna, L. Q. Chen, C. Holcomb, and A. D. Wasan. 2010. "Substance Misuse Treatment for High-Risk Chronic Pain Patients on Opioid Therapy: A Randomized Trial." Pain 150 (September): 390-400.

Liebschutz, J. M., Z. Xuan, C. W. Shanahan, M. LaRochelle, J. Keosaian, D. Beers, G. Guara, K. O’Connor, D. P. Alford, V. Parker, R. D. Weiss, J. H. Samet, J. Crosson, P. A. Cushman, and K. E. Lasser. 2017. "Improving Adherence to Long-term Opioid Therapy Guidelines to Reduce Opioid Misuse in Primary Care: A Cluster-Randomized Clinical Trial." JAMA Internal Medicine 177 (September): 1265-1272. 
Martel, M. O., A. J. Dolman, R. R. Edwards, R. N. Jamison, and A. D. Wasan. 2014. “The Association between Negative Affect and Prescription Opioid Misuse in Patients with Chronic Pain: The Mediating Role of Opioid Craving." The Journal of Pain 15 (January): $90-100$.

Michna, E., R. N. Jamison, L. D. Pham, E. L. Ross, D. Janfaza, S. S. Nedeljkovic, S. Narang, D. Palombi, and A. D. Wasan. 2007. "Urine Toxicology Screening among Chronic Pain Patients on Opioid Therapy: Frequency and Predictability of Abnormal Findings." The Clinical Journal of Pain 23 (February): 173-179.

National Academies of Sciences, Engineering, and Medicine. 2017. Pain Management and the Opioid Epidemic: Balancing Societal and Individual Benefits and Risks of Prescription Opioid Use. Washington, DC: The National Academies Press.

Quinones, S. 2015. Dreamland: The True Tale of America's Opiate Epidemic. New York: Bloomsbury Press.

Smith, S. M., F. Paillard, A. McKeown, L. B. Burke, R. R. Edwards, N. P. Katz, E. J. Papadopoulos, B. A. Rappaport, A. Slagle, E. C. Strain, A. D. Wasan, D. C. Turk, and R. H. Dworkin. 2015. "Instruments to Identify Prescription Medication Misuse, Abuse, and Related Events in Clinical Trials: An ACTTION Systematic Review." The Journal of Pain 16 (May): 389-411.

Tayeb, B. O., A. E. Barreiro, Y. S. Bradshaw, K. K. Chui, and D. B. Carr. 2016. "Durations of Opioid, Nonopioid Drug, and Behavioral Clinical Trials for Chronic Pain: Adequate or Inadequate?" Pain Medicine 17 (November): 2036-2046.

Vowles, K. E., M. L. McEntee, P. S. Julnes, T. Frohe, J. P. Ney, and D. N. van der Goes. 2015. "Rates of Opioid Misuse, Abuse, and Addiction in Chronic Pain: A Systematic Review and Data Synthesis." Pain 156 (April): 569-576.

Wasan, A. D., E. Michna, R. R. Edwards, J. N. Katz, S. S. Nedeljkovic, A. J. Dolman, D. Janfaza, Z. Isaac, and R. N. Jamison. 2015. "Psychiatric Comorbidity Is Associated Prospectively with Diminished Opioid Analgesia and Increased Opioid Misuse in Patients with Chronic Low Back Pain.” Anesthesiology 123 (October): 861-872.

Ajay D. Wasan is the vice chair for pain medicine in the Department of Anesthesiology at the University of Pittsburgh Medical Center (UPMC) and a professor of anesthesiology and psychiatry in the University of Pittsburgh School of Medicine. He is board certified in pain medicine and completed fellowship training in pain medicine in the Anesthesiology Department at Brigham and Women's Hospital and Harvard Medical School. He is also board certified in psychiatry and completed a residency in psychiatry at Johns Hopkins. He is cochair of a systemwide committee to improve pain care across UPMC. 